\title{
Laboratory Test Toxicity
}

National Cancer Institute

\section{Source}

National Cancer Institute. Laboratory Test Toxicity. NCI Thesaurus. Code C83323.

A laboratory test designed to quantify or qualify the poisonous nature of a sample. 